\title{
A Computer Aided Kineto-Static Analysis Method for 3-RRRT Parallel Manipulator Based on Vector Bond Graph
}

\author{
Zhongshuang Wang ${ }^{*}$ and Yangyang Tao
}

\author{
School of Mechatronics Engineering, Qiqihar University, Qiqihar, Heilongjiang 161006, China
}

\begin{abstract}
For improving the reliability and efficiency of the kineto-static analysis of complex robot systems, the corresponding vector bond graph procedure is proposed. By the kinematic constraint condition, the vector bond graph model of universal joint are made. Based on this, the vector bond model of 3-RRRT parallel Manipulator is built. For solving the algebraic difficulties brought by differential causality in system automatic kineto-static analysis, the effective bond graph augment method is proposed. From the algebraic relations of input and output vectors in the basic fields, junction structure and Euler-junction structure of system vector bond graph model, the unified formulae of driving moment (or force) and constraint forces at joints are derived, which are easily derived on a computer in a complete form. As a result, the automatic kineto-static analysis of 3-RRRT parallel Manipulator is realized, the validity of the method presented here is illustrated.
\end{abstract}

Keywords: 3-RRRT Parallel Manipulator, Kineto-Static Analysis, Vector Bond Graph, Causality, Bond Graph Augment, Constraint Joint.

\section{INTRODUCTION}

Parallel manipulators have been widely used in industry, such as machine tool, simulator for aircraft driver, automatic assembling for automobile production, and micromechanism for computer assisted surgery [1, 2], 3-RRRT parallel manipulator is one of important type of such systems. The kineto-static analysis is very important for the design, control, static and dynamic strength check of such system. Due to high nonlinearities and couplings involved in parallel manipulators, determining driving moment (or force) and the constraint forces at joints is a very tedious and erroprone task. The Newton-Euler technique and Lagrange technique are two of the well known methods used for the dynamic analysis of a robot system $[1,2]$. These techniques however, are only suitable for a single energy domain systems, e.g. mechanical systems, and can not be used to tackle systems that simultaneously include various physical domains in a unified manner.

The bond graph technique developed since the 1960's has potential applications in analyzing such complex systems and has been used successfully in many areas $[3,4]$. It is a pictorial representation of the dynamics of the system and clearly depicts the interaction between elements, it can also model multi-energy domains, for example, the actuator systems, which may be electrical, electro-magnetic, pneumatic, hydraulic or mechanical. Once the bond graph model of the system is ready, the system dynamic equations can be derived from it algorithmically in a systematic manner. This

*Address correspondence to this author at the School of Mechatronics Engineering, Qiqihar University, Qiqihar, Heilongjiang 161006, China;

E-mail:wzhsh1962@163.com process is usually automated by using appropriated software $[3,4]$. But for spatial multibody systems such as parallel manipultors with different constraint joints, the kinematic and geometric constraints between bodies result in differential causality loop, and the nonlinear velocity relationship between the mass center and joint point on a body leads to the nonlinear junction structure. The traditional bond graph procedures mentioned above were found to be very difficult algebraically in automatic modeling and kineto-static analysis of systems on a computer. To solve this problem, the Lagrange multiplier approach and Karnopp-Margolis approach $[5,6]$ can be employed to develop bond graph model for multibody systems. Based on the Karnopp-Margolis approach, Zeid proposed a new singularly perturbed explicit modeling method [7] and provided a more realistic joint bond graph model for revolute joint, prismatic joint, spherical joint, universal joint, and so forth based on scalar bond graph concept [8].

The bond graph technique and causality concept have been well developed for systems in which components have scalar constitutive laws. But for spatial multibody systems, the scalar bond graph technique is found to be complex, difficult and requires a great amount of experience, because three dimensional motion of system components must be resolved into scalar bonds. To address this problem, the vector bond graph techniques [9-11] were proposed. In vector bond graphs, single power bonds are replaced by multipower bonds, this makes it posses more concise presentation manner and be more suitable for modelling spatial multibody systems. Some problems however, should be studied further, such as modeling complex spatial robot mechanism with different constraint joints by vector bond graphs, augmenting the vector bond model to avoid differential causality, developing the generic algorithm for automatic kineto-static 


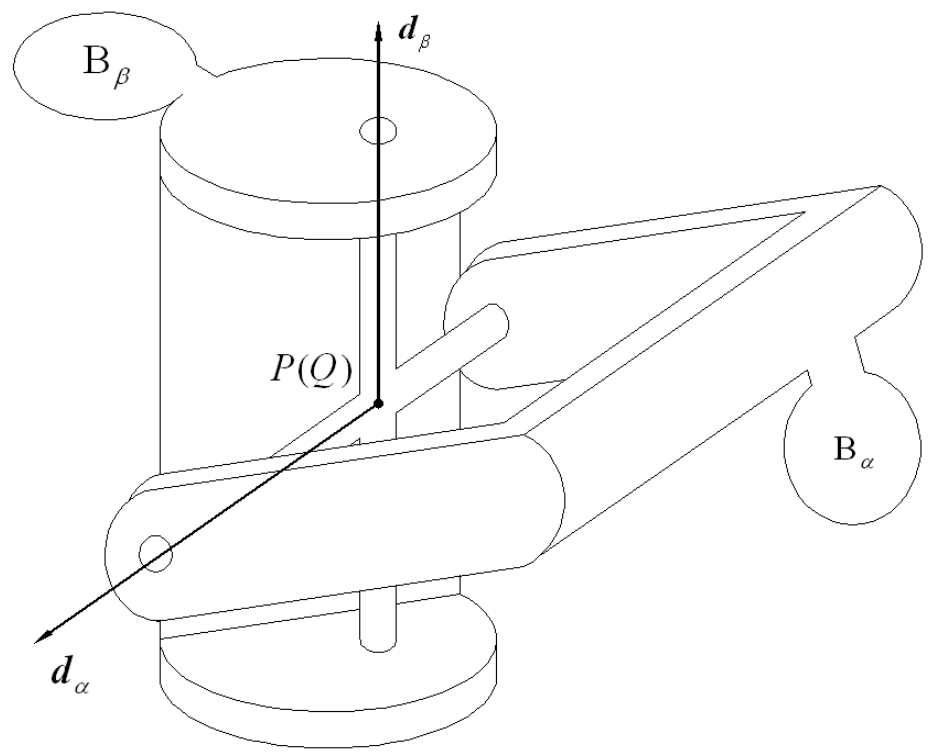

Fig. (1). The diagram of spatial universal joint.

analysis of spatial robot mechanism. To solve these problems, a more efficient and practical computer aided kinetostatic analysis procedure for complex spatial robot mechanism based on vector bond graph is proposed here.

\section{THE VECTOR BOND GRAPH MODEL OF UNI- VERSAL JOINT}

The diagram of spatial universal joint is shown in Fig. (1).

This joint allows only two direction rotations between its joined body $\mathrm{B}_{\alpha}$ and $\mathrm{B}_{\beta}$, fixing the remaining three translational and one rotational degrees of freedom. Therefore, only two generalized coordinates are free to change. Joint point $\mathrm{P}$ and $\mathrm{Q}$ are fixed on rigid body $\mathrm{B}_{\alpha}$ and $\mathrm{B}_{\beta}$ respectively, vector $h$ is used to describe the relative motion of the two rigid bodies, $h=r_{\alpha}^{P}-r_{\beta}^{Q}$. Where $r_{\alpha}^{P}$ and $r_{\beta}^{Q}$ represent the position vector of joint point $\mathrm{P}$ and $\mathrm{Q}$ in global coordinates respectively, $r_{\dot{a}}^{P}=\left[\begin{array}{lll}x_{\dot{a}}^{P} & y_{\dot{a}}^{P} & z_{\dot{a}}^{P}\end{array}\right]^{\mathrm{T}}, r_{\hat{a}}^{Q}=\left[\begin{array}{ll}x_{\hat{a}}^{Q} & y_{\hat{a}}^{Q}\end{array}\right.$ $\left.z_{\hat{a}}^{Q}\right]^{\mathrm{T}} \cdot d_{\alpha}$ and $d_{\beta}$ are two unit vectors fixed on rigid body $\mathrm{B}_{\dot{a}}$ and $\mathrm{B}_{\beta}$, which are parallel to axis, and orthogonal to each other. $d_{\alpha}$ and $d_{\beta}$ are the corresponding vectors in body frame. From the kinematic constraint condition of universal joint [1], the position constraint equations can be written as

$$
\begin{aligned}
& \underline{\Phi}^{(u)}\left(h, d_{\alpha}, d_{\beta}\right)=\left[\begin{array}{c}
r_{\alpha}^{P}-r_{\beta}^{Q} \\
d_{\beta}^{\mathrm{T}} d_{\alpha}
\end{array}\right] \\
& =\left[\begin{array}{c}
r_{\alpha}^{P}-r_{\beta}^{Q} \\
d_{\beta}^{\mathrm{T}} A^{\beta \mathrm{T}} A^{\alpha} d_{\alpha}^{\prime}
\end{array}\right]=0
\end{aligned}
$$

where $A^{\alpha}$ and $A^{\beta}$ are the direction cosin matrices of body $\mathrm{B}_{\alpha}$ and body $\mathrm{B}_{\beta}$ respectively.

The corresponding velocity (angular velocity) constraint equations can be written as

$$
\begin{aligned}
& \underline{\Phi}^{(u)}\left(h, d_{\alpha}, d_{\beta}\right)= \\
& \dot{\Phi}^{(u)}\left(h, d_{\alpha}, d_{\beta}\right)= \\
& {\left[\begin{array}{c}
\dot{r}_{\alpha}^{P}-\dot{r}_{\beta}^{Q} \\
d_{\beta}^{\mathrm{T}}\left(\tilde{d}_{\alpha}\right) \omega_{\alpha}+d_{\alpha}^{\mathrm{T}}\left(\tilde{d}_{\beta}\right) \omega_{\beta}
\end{array}\right]=0}
\end{aligned}
$$

where $d_{n}=A^{n} d_{n}^{\prime}, d_{n}=A^{n} d_{n}^{\prime}, \omega_{\dot{a}}$ and $\omega_{\hat{a}}$ represent the angular velocity vectors of the rigid body $B_{a}$ and $B_{\hat{a}}$ determined in global coordinates, $\omega_{\dot{a}}^{b}$ and $\omega_{\hat{a}}^{b}$ are the corresponding angular velocity vectors of the rigid body determined in body frame.

$$
\tilde{d}_{\alpha}=\left[\begin{array}{ccc}
0 & -d_{\alpha z} & d_{\alpha y} \\
d_{\alpha z} & 0 & -d_{\alpha x} \\
-d_{\alpha y} & d_{\alpha x} & 0
\end{array}\right], \tilde{d}_{\beta}=\left[\begin{array}{ccc}
0 & -d_{\beta z} & d_{\beta y} \\
d_{\beta z} & 0 & -d_{\beta x} \\
-d_{\beta y} & d_{\beta x} & 0
\end{array}\right]
$$

The velocity and angular velocity constraint equations shown in Eq. (2) can be presented by vector bond model shown in Fig. (2), where the modulus matrices of MTF can be obtained from Eq.(2) directly.

\section{THE VECTOR BOND MODEL OF 3-RRRT PAR- ALLEL MANIPULATOR}

As shown schematically in Fig. (3), a 3-RRRT parallel manipulator consists of a movable upper platform $(\Delta a b c)$, a fixed base platform $(\triangle A B C)$ and three parallel moving chains (A-a, B-b, and C-c). Each chain consists of three rigid 

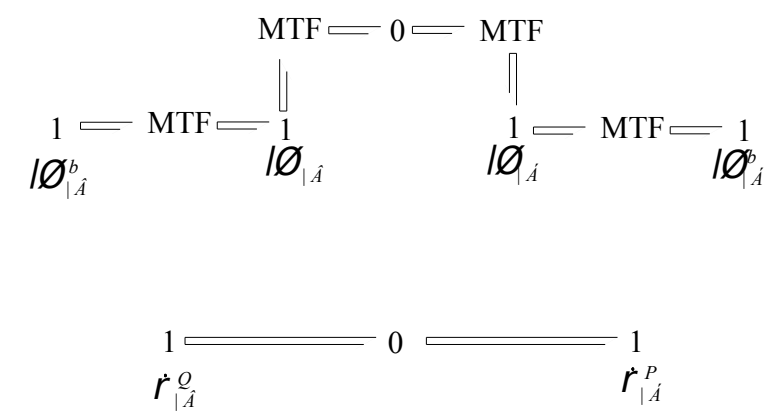

Fig. (2). The vector bond graph model of spatial universal joint.

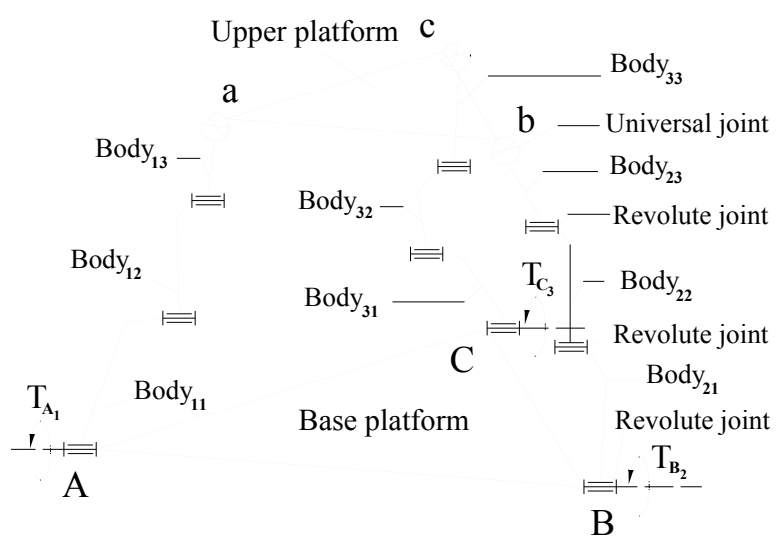

Fig. (3). The diagram of 3-RRRT manipulator.

bodies and is connected to the base and upper platform by a universal joint and a revolute joint respectively, and three bodies in a single moving chain are joined each other by two revolute joints. The joined structure of this system is shown in Fig. (4). For chain A-a, B-b and C-c, the corresponding driving moments are $T_{A_{1}}, T_{B_{2}}$, and $T_{\mathrm{C}_{3}}$ respectively.

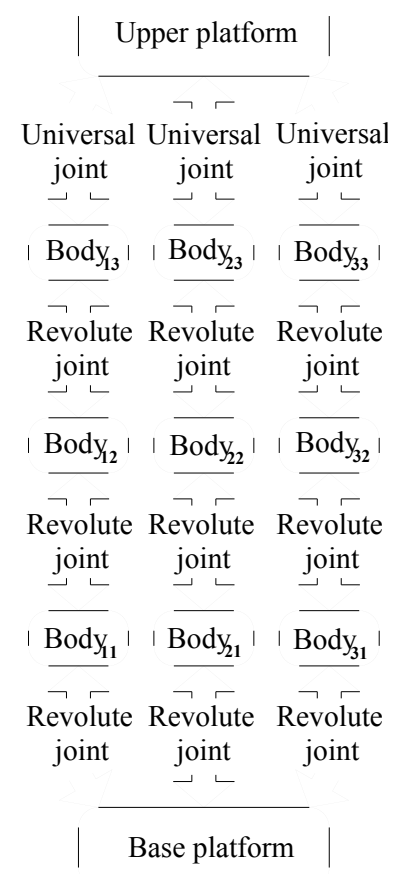

Fig. (4). The joined structure of 3-RRRT manipulator.
Fig. (5) shows the configuration of 3-RRRT manipulator which only contains a moving chain A-a for simplification. Global coordinates $C_{b p} X_{0} Y_{0} Z_{0}$ is located at the equilateral triangle center $C_{b p}$ of base platform, and body frame $C_{u p} X_{p} Y_{p} Z_{p}$ is located at the equilateral triangle center $C_{u p}$ of upper platform. The other body frames is located at the mass centers of bodies in three moving chains( A-a, B-b, C-c ). For example, $C_{a i} X_{a i} Y_{a i} Z_{a i}(i=1,2,3)$ represents the body frame corresponding to body $i$ in chain A-a. The circumradius of upper platform is $r$, and $R$ presents the circumradius of base platform. In Fig. (5), $\grave{e}_{j i}$ and $\dot{e}_{j i}$ represent relative angular displacement and relative angular velocity of body $i$ in chain $j$ respectively ( $i=1,2,3, j=1,2,3)$. If the motion of upper platform center $x_{u p}=f_{1}(t), y_{u p}=f_{2}(t)$ and $z_{u p}=f_{3}(t)$ are given, $\grave{e}_{j i}$ and $\dot{e}_{j i}$ can be determined by the kinematic analysis of mechanism [2]. Obviously, This manipulator has three degrees of freedom.

From the kinematic relations of mass center velocity vector, joint velocity vector in global coordinates and angular velocity vector in body frame, a spatial moving rigid body can be modeled by vector bond graph [11]. The NewtonEuler equation describing the rotation of a rigid body contains a term with an exterior product between angular momentum vector and angular velocity vector, this term can be expressed by vector bong graph and be called Euler-junction 


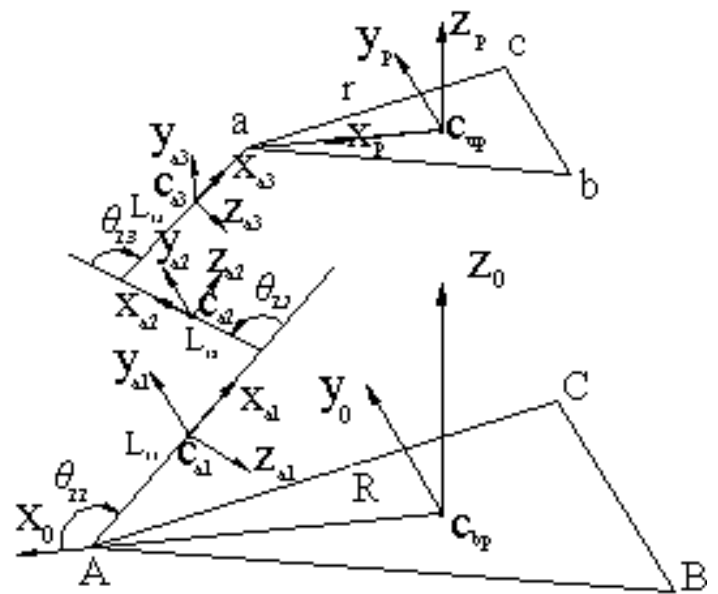

Fig. (5). The configuration of 3-RRRT manipulator.

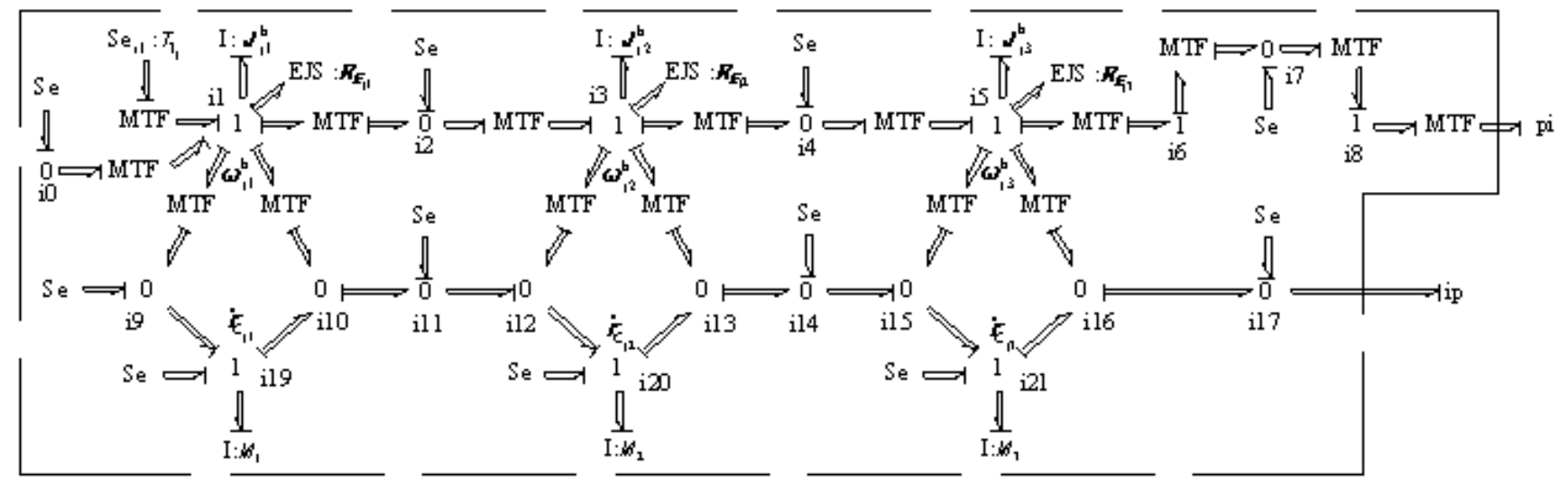

Fig. (6). The vector bond model of moving chain A-a, B-b, and C-c in 3-RRRT manipulator.

structure (EJS) $[11,12]$. For a spatial moving rigid body $i$ in chain $j(i=1,2,3, j=1,2,3)$, the relationship between input vector and output vector of its EJS can be written as

$$
E_{\text {out }_{j i}}=R_{E_{j i}}\left(\omega_{j i}^{\mathrm{b}}\right) \omega_{j i}^{\mathrm{b}}=R_{E_{j i}}\left(\omega_{j i}^{\mathrm{b}}\right) E_{i n_{j i}}
$$

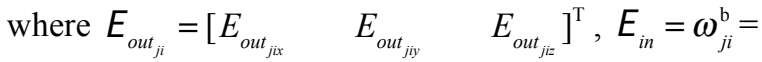

$\left[\omega_{j i x}^{\mathrm{b}} \omega_{j i y}^{\mathrm{b}} \omega_{j i z}^{\mathrm{b}}\right]^{\mathrm{T}}$. The angular velocity $\omega_{j i}^{\mathrm{b}}$ and moment of inertia matrix $J_{j i}^{\mathrm{b}}$ are both determined in body frame, $J_{j i}^{\mathrm{b}}=$ $\operatorname{diag}\left(J_{j i x}^{\mathrm{b}} J_{j i y}^{\mathrm{b}} J_{j i z}^{\mathrm{b}}\right)$.

$$
R_{E_{j i}}\left(\omega_{j i}^{\mathrm{b}}\right)=\left[\begin{array}{ccc}
0 & J_{j i z}^{\mathrm{b}} \omega_{j i z}^{\mathrm{b}} & -J_{j i y}^{\mathrm{b}} \omega_{j i y}^{\mathrm{b}} \\
-J_{j i z}^{\mathrm{b}} \omega_{j i z}^{\mathrm{b}} & 0 & J_{j i x}^{\mathrm{b}} \omega_{j i x}^{\mathrm{b}} \\
J_{j i y}^{\mathrm{b}} \omega_{j i y}^{\mathrm{b}} & -J_{j i x}^{\mathrm{b}} \omega_{j i x}^{\mathrm{b}} & 0
\end{array}\right]
$$

The spatial revolute joint allows turning the bodies joined between them. Therefore, three translations and two rotational degrees of freedom are constrained, leaving only one rotation degree of freedom free. For a single moving chain $j$ ( $j=1,2,3$, representing chain $\mathrm{A}-\mathrm{a}, \mathrm{B}-\mathrm{b}$, or $\mathrm{C}-\mathrm{c}$ respectively), the vector bond graph model can be made by assembling the vector bond graph of a single spatial moving rigid body [11], the revolute joints, and the universal joint, which is shown in Fig. (6).

In Fig. (6), if $i=\mathrm{a}$ and $j=1$, it presents the vector bond graph of moving chain $\mathrm{A}-\mathrm{a}$, if $i=\mathrm{b}$ and $j=2$, it presents the vector bond graph of moving chain B-b, and if $i=\mathrm{c}$ and $j=3$, it presents the vector bond graph of moving chain C-c. $\dot{r}_{C_{i i}}$ $(i=1,2,3, j=1,2,3)$ is the mass center velocity vector of body $i$ of chain $j$ in global coordinates, $\omega_{j i}^{\mathrm{b}}$ is the angular velocity vector of body $i$ of chain $j$ in body frame, $\omega_{j i}$ is the angular velocity vector of body $i$ of chain $j$ in global coordinates. The mass of body $i$ is $m_{C_{i}}, M_{i}=\operatorname{diag}\left(m_{C_{i}} m_{C_{i}} m_{C_{i}}\right)$. The vector bond graph shown in Fig. (6) can be simplified and shown in Fig. (7), where pi and ip are two output ports which connect the vector bond graph models of three moving chains (A-a, $\mathrm{B}-\mathrm{b}$, and $\mathrm{C}-\mathrm{c}$ ) to upper platform vector bond graph model ( $i=\mathrm{a}, \mathrm{b}, \mathrm{c}$ ). The upper platform is a spatial moving rigid body, its vector bond graph model is shown in Fig. (8), where $\dot{r}_{u p}$ is the mass center velocity vector of upper platform in global coordinates, The angular velocity vector $\omega_{u p}^{\mathrm{b}}$ 


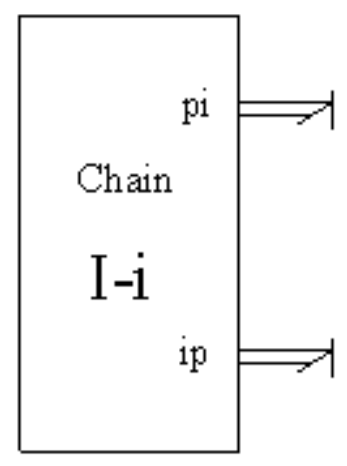

Fig. (7). The simplified model of Fig. (6).

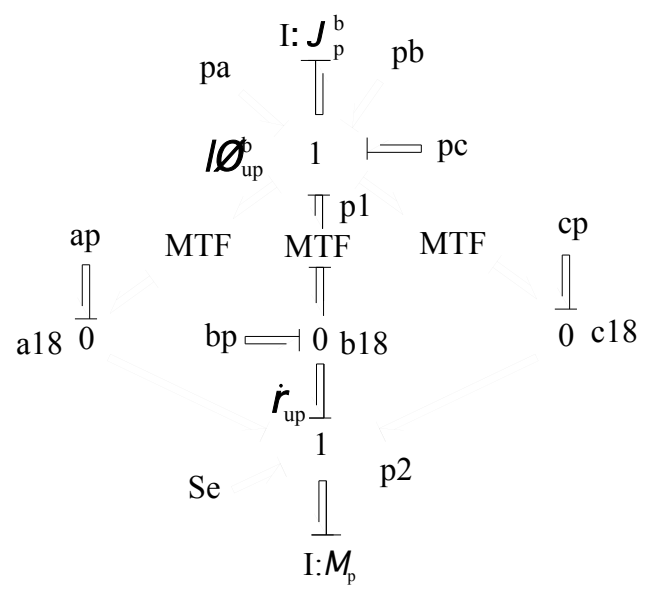

Fig. (8). The vector bond graph model of upper platform.

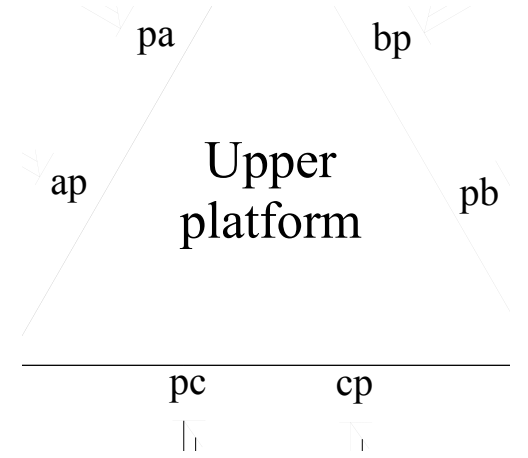

Fig. (9). The simplified vector bond graph model of upper platform.

and moment of inertia matrix $J_{p}^{\mathrm{b}}$ are both determined in body frame, $J_{p}^{\mathrm{b}}=\operatorname{diag}\left(J_{p x}^{\mathrm{b}} J_{p y}^{\mathrm{b}} J_{p z}^{\mathrm{b}}\right)$, the mass of upper platform is $m_{p C}, M_{p}=\operatorname{diag}\left(\begin{array}{lll}m_{p C} & m_{p C} & m_{p C}\end{array}\right)$.

The vector bond graph model of upper platform can be simplified and shown in Fig. (9), where ap, bp, cp, pa, pb, pc present the output ports of the vector bond graph mode of moving chains ( A-a, B-b, C-c ) respectively.

As a result, the overall vector bond graph model of 3RRRT manipulator can be made and shown in Fig. (10). However, the kinematic constraints result in differential causalities, the current vector bond graph procedures [9-11] were found to be very difficult algebraically in deriving system driving moment (or force) and the constraint force equa-

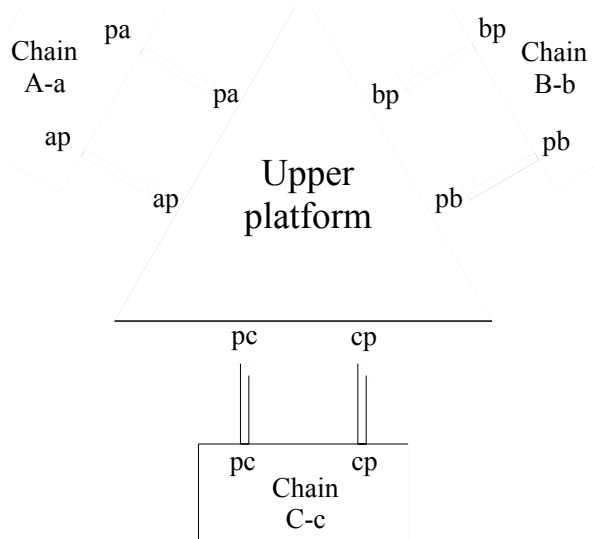

Fig. (10). The overall vector bond graph model of 3-RRRT manipulator.

tions automatically on a computer. To solve this problem, the constraint force (or moment ) vectors of joints can be considered as unknown source vectors, such as $\mathrm{Se}_{i 0}, \mathrm{Se}_{i 2}$, $S e_{i 4}, S e_{i 7}, S e_{i 9}, S e_{i l l}, S e_{i 14}, S e_{i 17}(\mathrm{i}=\mathrm{a}, \mathrm{b}, \mathrm{c})$, and added to the corresponding 0 -junctions. As a result, all differential causalities in this system vector bond graph can be eliminated, thus the procedure presented here can be used directly.

\section{THE UNIFIED FORMULAE OF DRIVING MO- MENT AND CONSTRAINT FORCES FOR SPATIAL ROBOT SYSTEMS}

The basic fields and junction structure of system bond graph is shown in Fig. (11) [3], where Euler-junction structure (EJS) $[11,12]$ is added. $X_{i_{1}}$ represents energy variable vector of independent storage energy field corresponding to independent motion, $X_{i_{2}}$ represents energy variable vector of independent storage energy field corresponding to dependent motion, $Z_{i_{1}}$ and $Z_{i_{2}}$ are the corresponding coenergy variable vectors. $D_{\text {in }}$ and $D_{\text {out }}$ represent input and output vector variables in resistive field, $U$ and $V$ represent input and output vector variables of source field respectively, $U=\left[\begin{array}{lll}U_{1} & U_{2} & U_{3}\end{array}\right]^{\mathrm{T}}, V=$

$\left[\begin{array}{lll}V_{1} & V_{2} V_{3}\end{array}\right]^{\mathrm{T}}$. Where $U_{1}$ is driving moment (or force) vector, $U_{2}$ is the constraint force vector of joint, and $U_{3}$ is known source vector. $E_{\text {in }}$ and $E_{\text {out }}$ are the input and output variable vectors in Euler-junction structure (EJS).

For independent energy storage field, we have

$\mathrm{Z}_{\mathrm{i}_{1}}=\mathrm{F}_{\mathrm{i}_{1}} \mathrm{X}_{\mathrm{i}_{1}}$

$Z_{i_{2}}=F_{i_{2}} X_{i_{2}}$

where $F_{i_{1}}$ and $F_{i_{2}}$ are $m_{1} \times m_{1}$ and $m_{2} \times m_{2}$ matrices respectively.

For resistive field, we have 
$D_{\text {out }}=R D_{\text {in }}$

where $R$ is $L \times L$ matrix.

The EJS equations of a overall multibody system can be assembled by Eq.(3) and written as

$$
E_{\text {out }}=R_{E} E_{\text {in }}
$$

where $E_{\text {out }}=\left[\begin{array}{lll}E_{\text {out }_{1}} & E_{\text {out }_{2}} \cdots E_{\text {out }_{n}}\end{array}\right]^{\mathrm{T}}, E_{\text {in }}=\left[\begin{array}{ll}E_{\text {in }} & E_{\text {in }} \\ \end{array}\right.$

$$
\left.\cdots E_{i n_{\mathrm{n}}}\right]^{\mathrm{T}}, R_{E}=\operatorname{diag}\left(R_{E_{1}} \quad R_{E_{2}} L R_{E_{n}}\right) \text {. }
$$

The corresponding junction structure equations can be written as

$$
\begin{aligned}
& \dot{X}_{i_{l}}=J_{i_{1} i_{1}} Z_{i_{1}}+J_{i_{1} i_{2}} Z_{i_{2}}+J_{i_{1} L} D_{\text {out }}+J_{i_{1} u_{I}} U_{1} \\
& +J_{i_{1} u_{2}} U_{2}+J_{i_{1} u_{3}} U_{3}+J_{i_{1} E} E_{\text {out }} \\
& \dot{X}_{i_{2}}=J_{i_{2} i_{1}} Z_{i_{1}}+J_{i_{2} i_{2}} Z_{i_{2}}+J_{i_{2} L} D_{o u t}+J_{i_{2} u_{1}} U_{1} \\
& +J_{i_{2} u_{2}} U_{2}+J_{i_{2} u_{3}} U_{3}+J_{i_{2} E} E_{o u t} \\
& D_{i n}=J_{L i_{1}} Z_{i_{1}}+J_{L i_{2}} Z_{i_{2}}+J_{L L} D_{\text {out }}+J_{L u_{1}} U_{1} \\
& +J_{L u_{2}} U_{2}+J_{L u_{3}} U_{3}+J_{L E} E_{o u t} \\
& E_{\text {in }}=J_{E i_{1}} Z_{i_{1}}+J_{E i_{2}} Z_{i_{2}}+J_{E L} D_{o u t}+J_{E u_{1}} U_{1} \\
& +J_{E u_{2}} U_{2}+J_{E u_{3}} U_{3}+J_{E E} E_{o u t}
\end{aligned}
$$

From the flow summation of 0 -junctions corresponding to $m_{2}$ constraint force vectors in system vector bond graph model, we have

$0=J_{C i_{1}} Z_{i_{1}}+J_{C i_{2}} Z_{i_{2}}+J_{C L} D_{\text {out }}+J_{C u_{3}} U_{3}$

$$
+J_{C E} E_{o u t}
$$

By the algebraic manipulation from Eq.(4) Eq.(12), the system driving moment and constraint force equations can be written as

$$
\begin{aligned}
& \text { If } J_{C L}=0, J_{C E}=0 \\
& \begin{array}{l}
U_{1}=S_{u_{1} u_{1}}^{-1}\left(S_{u_{1} i_{1}} X_{i_{1}}+S_{u_{1} i_{2}} X_{i_{2}}+S_{u_{1} u_{3}} U_{3}\right. \\
\left.+T_{i_{1} u_{1}}^{\mathrm{T}} \dot{X}_{i_{1}}+T_{i_{1} u_{1}}^{\mathrm{T}} T_{i_{1} u_{2}} H_{4}^{-1} J_{c u_{3}} \dot{U}_{3}\right) \\
\quad U_{2}=\left(-H_{4}\right)^{-1}\left(H_{1} X_{i_{1}}+H_{2} X_{i_{2}}+H_{3} U_{1}\right. \\
\left.\quad+H_{5} U_{3}+J_{c u_{3}} \dot{U}_{3}\right)
\end{array}
\end{aligned}
$$

where

$$
\begin{aligned}
& A_{1}=\left[I_{2}-J_{E L} R\left(I_{1}-J_{L L} R\right)^{-1} J_{L E} R_{E}-J_{E E} R_{E}\right]^{-1} \\
& A_{2}=J_{E i_{1}} F_{i_{1}}+J_{E L} R\left(I_{1}-J_{L L} R\right)^{-1} J_{L i_{1}} F_{i_{1}} \\
& A_{3}=J_{E i_{2}} F_{i_{2}}+J_{E L} R\left(I_{1}-J_{L L} R\right)^{-1} J_{L i_{2}} F_{i_{2}}
\end{aligned}
$$

$$
A_{4}=J_{L u_{1}}+J_{E L} R\left(I_{1}-J_{L L} R\right)^{-1} J_{L u_{1}}
$$$$
A_{5}=J_{E u_{2}}+J_{E L} R\left(I_{1}-J_{L L} R\right)^{-1} J_{L u_{2}}
$$$$
A_{6}=J_{E u_{3}}+J_{E L} R\left(I_{1}-J_{L L} R\right)^{-1} J_{L u_{3}}
$$$$
B_{1}=\left(I_{1}-J_{L L} R\right)^{-1}\left(J_{L i_{1}} F_{i_{1}}+J_{L E} R_{E} A_{1} A_{2}\right)
$$$$
B_{2}=\left(I_{1}-J_{L L} R\right)^{-1}\left(J_{L i_{2}} F_{i_{2}}+J_{L E} R_{E} A_{1} A_{3}\right)
$$$$
B_{3}=\left(I_{1}-J_{L L} R\right)^{-1}\left(J_{L u_{1}}+J_{L E} R_{E} A_{1} A_{4}\right)
$$$$
B_{4}=\left(I_{1}-J_{L L} R\right)^{-1}\left(J_{L u_{2}}+J_{L E} R_{E} A_{1} A_{5}\right)
$$$$
B_{5}=\left(I_{1}-J_{L L} R\right)^{-1}\left(J_{L u_{3}}+J_{L E} R_{E} A_{1} A_{6}\right)
$$$$
T_{i_{1} i_{l}}=J_{i_{l} i_{l}} F_{i_{l}}+J_{i_{1} L} R B_{1}+J_{i_{1} E} R_{E} A_{1} A_{2}
$$$$
T_{i_{1} i_{2}}=J_{i_{1} i_{2}} F_{i_{2}}+J_{i_{1} L} R B_{2}+J_{i_{1} E} R_{E} A_{1} A_{3}
$$$$
T_{i_{l} u_{I}}=J_{i_{L} L} R B_{3}+J_{i_{l} u_{I}}+J_{i_{I} E} R_{E} A_{1} A_{4}
$$$$
T_{i_{1} u_{2}}=J_{i_{1} L} R B_{4}+J_{i_{1} u_{2}}+J_{i_{1} E} R_{E} A_{1} A_{5}
$$$$
T_{i_{1} u_{3}}=J_{i_{1} L} R B_{5}+J_{i_{1} u_{3}}+J_{i_{1} E} R_{E} A_{1} A_{6}
$$$$
H_{1}=\dot{J}_{C i_{1}} F_{i_{1}}+J_{C i_{1}} F_{i_{1}} T_{i_{1} i_{1}}+J_{C i_{2}} F_{i_{2}} T_{i_{2} i_{1}}
$$$$
H_{2}=\dot{J}_{C_{2}} F_{i_{2}}+J_{C i_{1}} F_{i_{1}} T_{i_{1} i_{2}}+J_{C i_{2}} F_{i_{2}} T_{i_{2} i_{2}}
$$$$
H_{3}=J_{C i_{1}} F_{i_{1}} T_{i_{1} u_{1}}+J_{C i_{2}} F_{i_{2}} T_{i_{2} u_{1}}
$$$$
H_{4}=J_{C i_{1}} F_{i_{1}} T_{i_{1} u_{2}}+J_{C i_{2}} F_{i_{2}} T_{i_{2} u_{2}}
$$$$
H_{5}=\dot{J}_{C u_{3}}+J_{C i_{1}} F_{i_{1}} T_{i_{1} u_{3}}+J_{C i_{2}} F_{i_{2}} T_{i_{2} u_{3}}
$$$$
S_{u_{1} u_{1}}=T_{i_{1} u_{1}}^{\mathrm{T}}\left[T_{i_{1} u_{1}}+T_{i_{1} u_{2}}\left(-H_{4}\right)^{-1} H_{3}\right]
$$$$
S_{u_{1} i_{1}}=T_{i_{1} u_{1}}^{\mathrm{T}}\left(T_{i_{1} u_{2}} H_{4}^{-1} H_{1}-T_{i_{1} i_{1}}\right)
$$$$
S_{u_{1} i_{2}}=T_{i_{1} u_{1}}^{\mathrm{T}}\left(T_{i_{1} u_{2}} H_{4}^{-1} H_{2}-T_{i_{1} i_{2}}\right)
$$$$
S_{u_{1} u_{3}}=T_{i_{1} u_{l}}^{\mathrm{T}}\left(T_{i_{1} u_{2}} H_{4}^{-1} H_{5}-T_{i_{1} u_{3}}\right)
$$

If $J_{C L} \neq 0$ or $J_{C E} \neq 0$

$$
\begin{aligned}
& U_{1}=D_{u_{1} u_{1}}^{-1}\left(D_{u_{1} i_{1}} X_{i_{1}}+D_{u_{1} i_{2}} X_{i_{2}}+D_{u_{1} u_{3}} U_{3}\right. \\
& \left.+T_{i_{1} u_{1}}^{\mathrm{T}} \dot{X}_{i_{1}}\right) \\
& U_{2}=\left(-T_{C u_{2}}\right)^{-1}\left(T_{C i_{1}} X_{i_{1}}+T_{C i_{2}} X_{i_{2}}+T_{C u_{1}} U_{1}\right. \\
& \left.+T_{C u_{3}} U_{3}\right)
\end{aligned}
$$




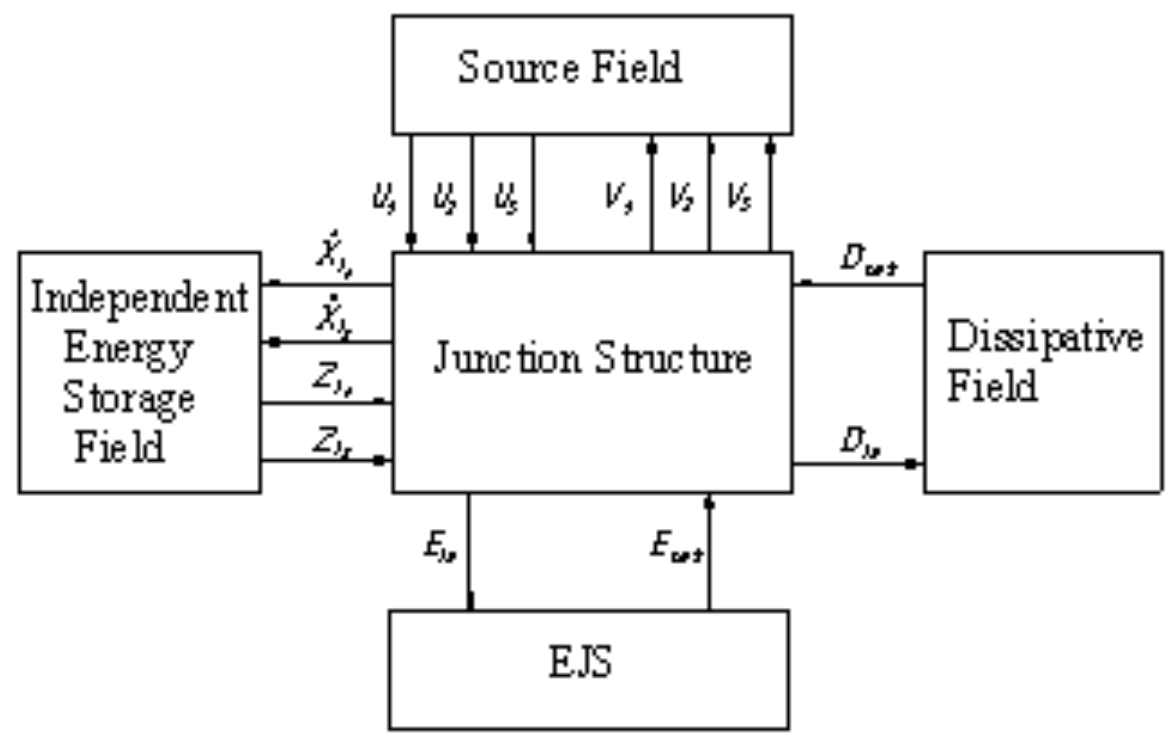

Fig. (11). The basic field and junction structure of system.

$$
\begin{aligned}
& T_{C i_{l}}=J_{C i_{l}} F_{i_{l}}+J_{C L} R B_{1}+J_{C E} R_{E} A_{1} A_{2} \\
& T_{C_{i_{2}}}=J_{C_{i_{2}}} F_{i_{2}}+J_{C L} R B_{2}+J_{C E} R_{E} A_{1} A_{3} \\
& T_{C u_{1}}=J_{C L} R B_{3}+J_{C E} R_{E} A_{1} A_{4} \\
& T_{\mathrm{Cu}_{2}}=J_{C L} R B_{4}+J_{C E} R_{E} A_{1} A_{5} \\
& T_{\mathrm{Cu}_{3}}=J_{C L} R B_{5}+J_{C u_{3}}+J_{C E} R_{E} A_{1} A_{6} \\
& D_{u_{l} u_{l}}=T_{i_{l} u_{I}}^{T}\left[T_{i_{l} u_{I}}+T_{i_{l} u_{2}}\left(-T_{C u_{2}}\right)^{-1} T_{C u_{l}}\right] \\
& D_{u_{1} i_{l}}=T_{i_{1} u_{l}}^{T}\left(T_{i_{l} u_{2}} T_{C u_{2}}^{-1} T_{C i_{I}}-T_{i_{1} i_{I}}\right) \\
& D_{u_{1} i_{2}}=T_{i_{1} u_{1}}^{T}\left(T_{i_{1} u_{2}} T_{C u_{2}}^{-1} T_{C_{2}}-T_{i_{1} i_{2}}\right) \\
& D_{u_{1} u_{3}}=T_{i_{1} u_{1}}^{T}\left(T_{i_{1} u_{2}} T_{C u_{2}}^{-1} T_{C u_{3}}-T_{i_{1} u_{3}}\right)
\end{aligned}
$$

Giving the system independent moving state variable vector $X_{i_{l}}$ and its derivative $\dot{X}_{i_{l}}$, the corresponding system driving moment (or force) vector $U_{1}$ and constraint force vector $U_{2}$ can be determined from Eq.(13) or Eq.(14) directly.

\section{KINETO-STATIC ANALYSIS EXAMPLE}

To check the model and procedure presented here, the kineto-static analysis for 3-RRRT manipulator is carried out. For chain $j(j=1,2,3)$, the mass of body $i(i=1,2,3)$ is $M_{j i}$, $\mathrm{M}_{j 1}=1 \mathrm{~kg}, \mathrm{M}_{j 2}=0.25 \mathrm{~kg}, \mathrm{M}_{j 3}=2 \mathrm{~kg}$. The length of body $i$ $(i=1,2,3)$ in chain $j(j=1,2,3)$ is presented $L_{j i}, \mathrm{~L}_{j 1}=0.4 \mathrm{~m}$, $\mathrm{L}_{j 2}=0.1 \mathrm{~m}, \mathrm{~L}_{j 3}=0.8 \mathrm{~m}$. $\mathrm{R}=0.4, \mathrm{r}=0.1 \mathrm{~m}$. The moment of inertia matrix is $J_{j i}^{\mathrm{b}}$.

$$
J_{j i}^{\mathrm{b}}=\left[\begin{array}{ccc}
\frac{1}{12} \mathrm{M}_{j i} L_{j i}^{2} & 0 & 0 \\
0 & \frac{1}{12} \mathrm{M}_{j i} L_{j i}^{2} & 0 \\
0 & 0 & \frac{1}{12} \mathrm{M}_{j i} L_{j i}^{2}
\end{array}\right]
$$

The mass center motion of upper platform are as following, $x_{u p}=0.05 \cos (0.5 \partial t), y_{u p}=0.05 \sin (0.5 \partial t), z_{u p}=0.8$.

From Fig. (10), vector $X_{i_{1}}, X_{i_{2}}, Z_{i_{1}}, Z_{i_{2}}, D_{\text {in }}, D_{\text {out }}, U_{1}$, $U_{2}, U_{3}, V_{1}, V_{2}, V_{3}, D_{\text {in }}, D_{\text {out }}, E_{\text {in }}$ and $E_{\text {out }}$ can be defined. By Eq.(4) $\sim$ Eq.(7), the matrix $F_{i_{l}}, F_{i_{2}}, R$ and $R_{E}$ can be obtained.

Also the coefficient matrices of Eq. (8) Eq.(12) which are called junction structure matrices can be got from Fig. (10). In this example, because the motion of upper platform is in a plane, so that the corresponding EJS can be eliminated shown as Fig. (8).

Inputting the physical parameters of the manipulator, the matrix $F_{i_{l}}, F_{i_{2}}, R$ and junction structure matrices in Eq.(8) Eq.(12), known source vector $U_{3}$, system independent moving state variable vector $X_{i_{I}}$, and its derivative $\dot{X}_{i_{1}}$ into the program associated with the procedure presented here based on MATLAB [13], the system driving moment (or force) and constraint force equations in the form of Eq. (14) can be derived on a computer, and the corresponding driving moment (or force) and constraint forces can be determined. Some of results are shown in (Fig. 12 to 17).

For this example, the Newton-Euler method [1] was used to determine the corresponding driving moment (or force) and constraint forces, the results are in good agreement with that obtained by the procedure in this paper. However, this process is very labor-intensive and tedious. 


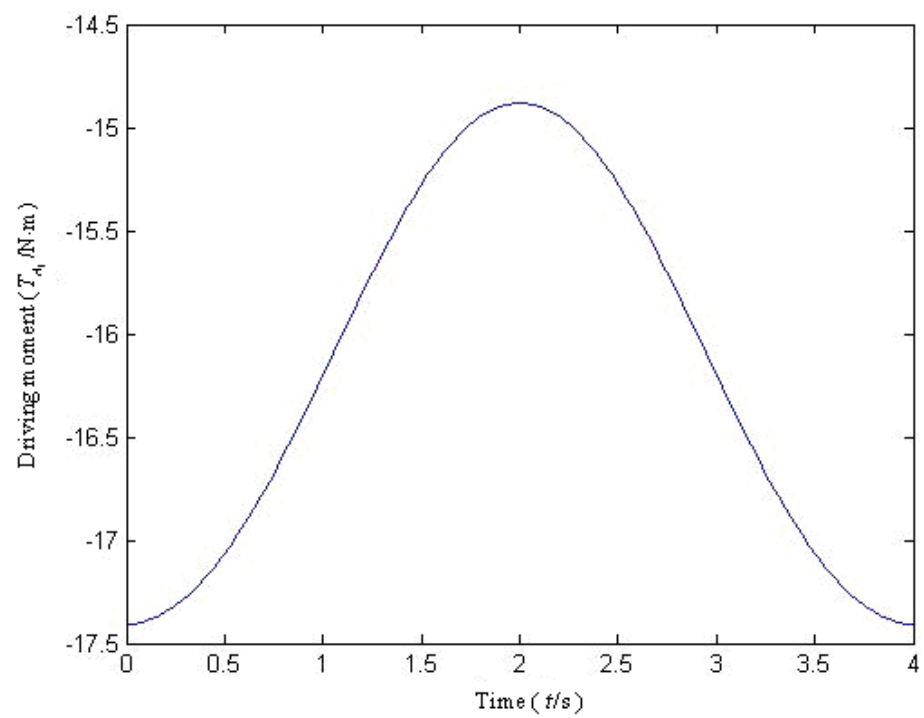

Fig. (12). The driving moment of chain A-a.

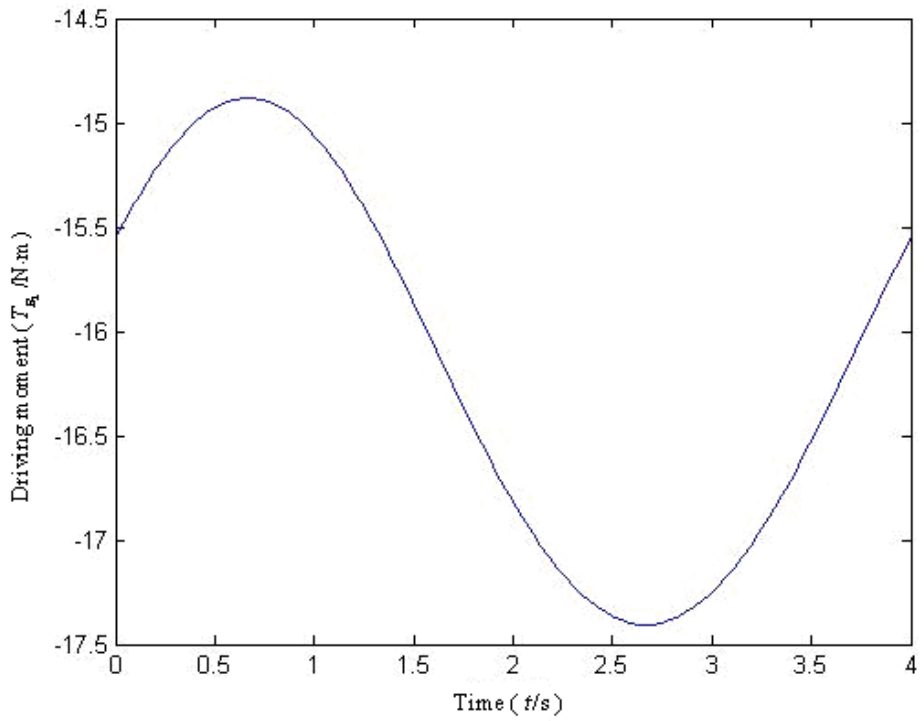

Fig. (13). The driving moment of chain B-b.

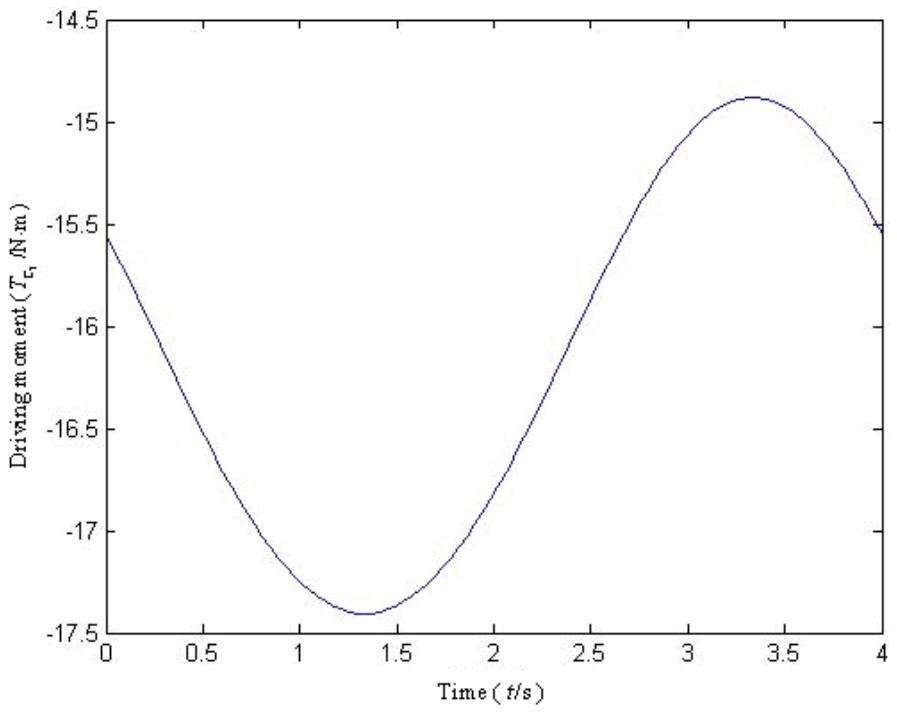

Fig. (14). The driving moment of chain C-c. 


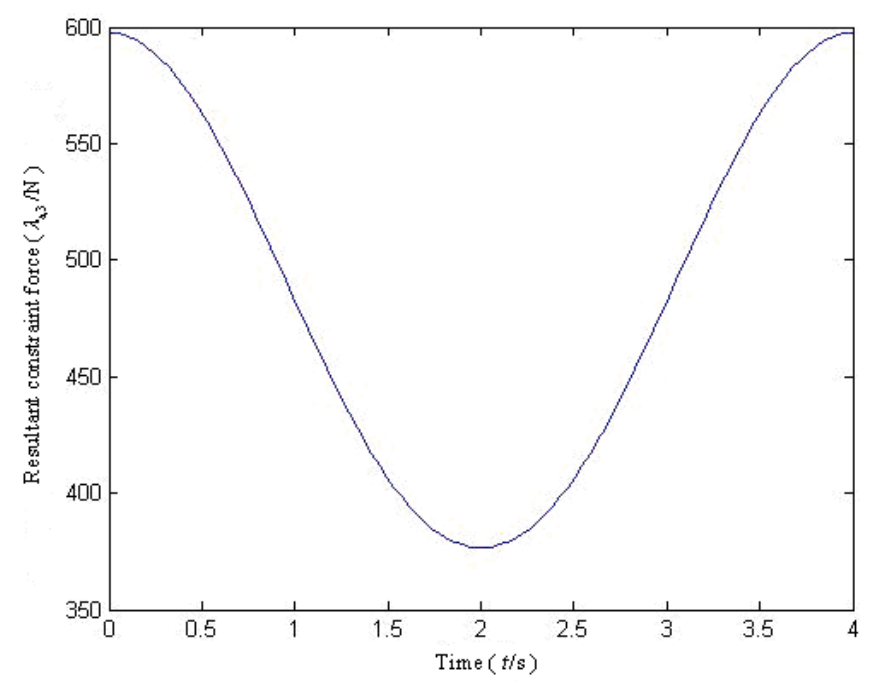

Fig. (15). Resultant constraint force between upper platform and body 3 in chain A-a.

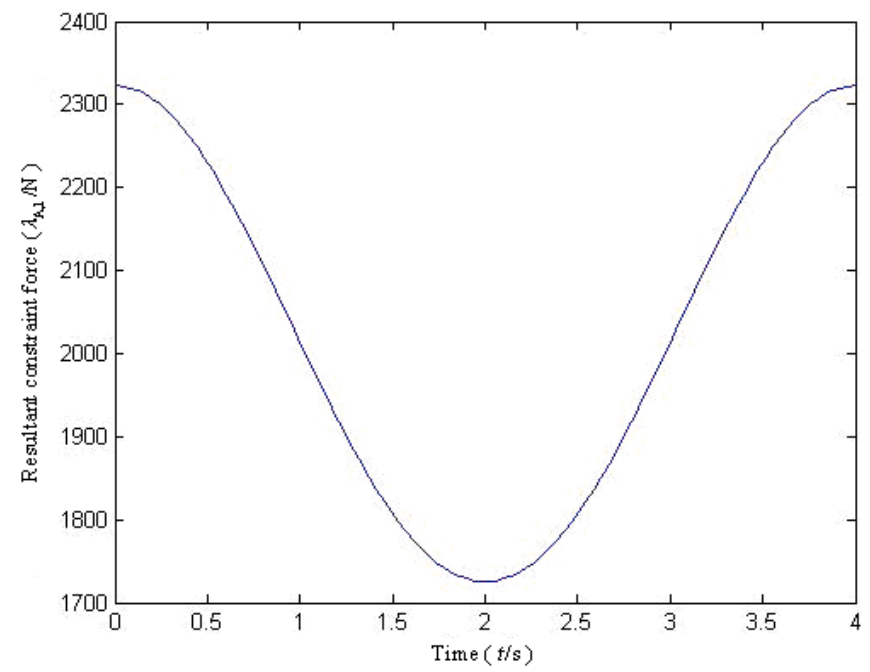

Fig. (16). Resultant constraint force between base platform and body 1 in chain A-a.

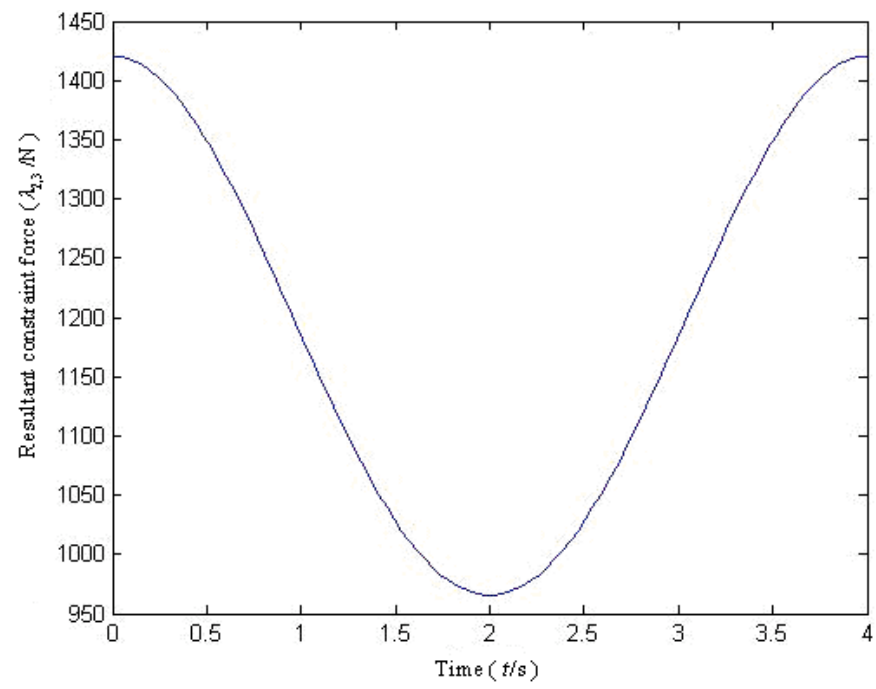

Fig. (17). Resultant constraint force between body 2 and body 3 in chain A-a.

From the descriptions above, the method presented here is suitable for both open loop robot mechanism and closed loop robot mechanism. Besides this, the method is not required mechanism acceleration analysis at all. Thus the reliability and efficiency of the kineto-static analysis of complex robot systems can be improved by the procedure presented here. 


\section{CONCLUSIONS}

The vector bond graph procedure presented here is very suitable for dealing with computer aided kineto-static analysis of complex robot systems with the coupling of multienergy domains. Compared with traditional scalar bond graph method, this vector bond graph procedure is more suitable for complex spatial robot mechanism because of its more compact and concise representation manner. The differential causalities in the vector bond graph model of spatial robot mechanisms can be avoided by the bond graph augment method proposed here, thus the algebraic difficulties in system automatic modeling and kineto-static analysis can be overcome. In the case of considering EJS, the unified formulae of system driving moment and constraint force equations are derived, which are easily derived on a computer in a complete form. Besides these, the procedure is suitable for both open loop robot mechanism and closed loop robot mechanism and not required mechanism acceleration analysis at all. These lead to a more efficient and practical automated procedure for kineto-static analysis of complex robot systems over a multi-energy domains in a unified manner. The validity of the procedure is illustrated by successful application to the kineto-static analysis of 3-RRRT manipulator.

\section{CONFLICT OF INTEREST}

The authors confirm that this article content has no conflicts of interest.

\section{ACKNOWLEDGMENTS}

This work was financially supported by National Natural Science Foundation of China (Grant No. 51175272).

\section{REFERENCES}

[1] J. Z. Hong, Computational Dynamics of Multibody Systems, The Advanced Education Press, Beijing, 2003.

[2] Z. Huang, L. F. Kong and Y. F. Fang, The parallel robot mechanism theory and control, Machine Press, Beijing, 1997.

[3] D. C. Karnopp, D. L. Margolis and R. C. Rosenberg, System dynamics: modeling and simulation of mechatronic systems, 4th ed., John Wiley, New York, 2006

[4] R. C. Rosenberg, "Reflections on Engineering Systems and Bond Graphs", ASME, J. Dyn. Syst, Meas. Control, vol. 115, no. 2, pp. 242-251, 1993.

[5] D. C. Karnopp, "Understanding multibody dynamics using bond graph representations", J. Franklin. Inst., vol. 334B, no. 4, pp. 631642, 1997.

[6] D. L. Margolis and D. C. Karnopp, "Bond Graph for Flexible Multibody Systems", Trans. ASME Ser. G, vol. 101, no.1, pp. 50$51,1979$.

[7] A. A. Zeid, J. L. Overholt, "Singularly perturbed bond graph models for simulation of multibody systems", J. Dyn. Syst., Meas. Control., vol.117, no.3, pp. 401-410, 1995

[8] Zeid, C. H. Chung, "Bond graph modelling of multibody systems: a library of three-dimensional joints", J. Franklin. Inst., vol. 329, no. 4, pp. 605-636, 1992.

[9] J. Jinhee, H. Changsoo, "Proposion of a modelling method for constrained mechanical systems based on vector bond graphs", $J$. Franklin. Inst., vol. 335B, no. 3, pp. 451-469, 1998.

[10] F. Wilfird, S. Serge, "Bond Graph Representation of Multibody Systems with Kinematic Loops", J. Franklin. Inst., vol. 335, no. 4 , pp. 643-650, 1998.

[11] S. Behzadipour, A. Khajepour, "Causality in vector bond graphs and its application to modeling of multi-body dynamic systems", Simul. Modell. Prac. Theory, vol. 14, pp. 279-295, 2006.

[12] P. Breedveld, "Stability of Rigid Rotation from a Bond Graph Pespective", Simul. Modell. Prac. Theory, vol.17, pp. 92-106, 2009.

[13] J. F. Jiang, L. J. Hu and J. Tang, Numerical Analysis and MATLAB Experiment, Science Press, Beijing, 2004

Received: August 13, 2013

(C) Wang and Tao; Licensee Bentham Open.

This is an open access article licensed under the terms of the Creative Commons Attribution Non-Commercial License (http://creativecommons.org/licenses/by-nc/3.0/) which permits unrestricted, non-commercial use, distribution and reproduction in any medium, provided the work is properly cited. 